\title{
Preferences for Adult Pneumococcal Vaccine Recommendations Among United States Health Care Providers
}

\author{
Patricia Sacco $\cdot$ Kelley Myers $\cdot$ Christine Poulos $\cdot$ Carolyn Sweeney \\ Kelly Hollis $\cdot$ Vincenza Snow $\cdot$ Jeffrey T. Vietri
}

Received: April 30, 2019 / Published online: September 23, 2019

(C) The Author(s) 2019

\begin{abstract}
Introduction: In 2014, the Advisory Committee on Immunization Practices (ACIP) of the US Centers for Disease Control and Prevention (CDC) recommended 13-valent pneumococcal conjugate vaccine (PCV13) followed by 23-valent pneumococcal polysaccharide vaccine (PPSV23) for all adults aged $\geq 65$ years, with a commitment to revisit the recommendation for PCV13 because of declining vaccine-type disease. The Evidence-to-Recommendation framework used by the ACIP includes review of evidence regarding feasibility and stakeholder acceptability, but no surveys of vaccinator preferences have been published in the literature.
\end{abstract}

Enhanced Digital Features To view enhanced digital features for this article go to https://doi.org/10.6084/ m9.figshare.9762098.

Electronic Supplementary Material The online version of this article (https://doi.org/10.1007/s40121019-00266-5) contains supplementary material, which is available to authorized users.

P. Sacco $(\varangle) \cdot$ K. Myers · C. Poulos · C. Sweeney · K. Hollis

RTI Health Solutions, Research Triangle Park, NC, USA

e-mail: psacco@rti.org

V. Snow $\cdot$ J. T. Vietri

Pfizer, Inc., New York, NY, USA
Methods: Physicians $(N=700), \quad$ physician assistants $(N=100)$, pharmacists $(N=100)$, and nurse practitioners $(N=100)$ who recently prescribed, administered, or recommended adult pneumococcal vaccine were surveyed in March 2018. Object-case best-worst scaling was used to assess preferences among potential recommendation scenarios: retaining the then-current 2014 recommendation without a scheduled re-evaluation, retaining with a scheduled reevaluation, revising PCV13 to Category B (retaining PPSV23 as Category A), removing PCV13 (retaining PPSV23 as Category A), and removing both PCV13 and PPSV23.

Results: Providers' most preferred recommendations were retaining the 2014 recommendation with another planned re-evaluation $(52.6 \%)$ and retaining the then-current recommendation without planned re-evaluation (40.0\%). Few preferred changing PCV13 to Category B (3.2\%), removing PCV13 (3.7\%), or removing both pneumococcal vaccines $(0.5 \%)$. Conclusions: The majority of vaccinators surveyed preferred to retain the 2014 recommendation, either with another scheduled reassessment or indefinitely.

Funding: Pfizer, Inc.

Keywords: Adult; Health care provider; Pneumococcal vaccine; Preferences 


\section{INTRODUCTION}

Streptococcus pneumoniae is a leading cause of serious illness, including bacteremia, meningitis, and pneumonia, among older adults in the US [1]. In the US, it is estimated that $>1$ million people will get pneumococcal disease each year, and the disease will cause thousands of deaths, particularly in older adults (aged 65 years and older) [2]. In 2014, the Advisory Committee on Immunization Practices (ACIP) of the US Centers for Disease Control and Prevention (CDC) issued a Category A recommendation for 13-valent pneumococcal conjugate vaccine (PCV13, Prevnar 13; Pfizer Inc., USA) for all US adults aged 65 years and older. This was added to the long-standing recommendation for use of 23-valent pneumococcal polysaccharide vaccine (PPSV23, Pneumovax; Merck and Co., Inc., USA) $[1,3]$. The vaccines were to be administered in series, with PCV13 given first and PPSV23 given at least 1 year later; for those who had previously received PPSV23 when aged $<65$ years and for whom an additional dose of PPSV23 is indicated when aged 65 years and older, a subsequent PPSV23 dose should be given $\geq 1$ year after PCV13 and $\geq 5$ years after the most recent dose of PPSV23 [4]. This recommendation included a planned re-evaluation of the use of PCV13 to be started in 2018, as the committee anticipated a reduction in vaccinepreventable adult disease due to indirect effects from the pediatric PCV13 program.

The ACIP recently adopted the Evidence to Recommendation (EtR) framework to review vaccine recommendations, including the reevaluation of use of PCV13 in adults aged 65 years and older [5]. This framework formalizes considerations that were only informally considered in the prior process, including stakeholder acceptability, feasibility, and preferences of the target population.

The objective of this study was to assess health care provider (HCP) preferences for potential changes to the pneumococcal disease immunization recommendation among adults aged 65 years and older using a methodologically robust approach as well as describe associated knowledge, attitudes, and beliefs.

\section{METHODS}

This study was a noninterventional, cross-sectional, web-based survey of primary care physicians, physician assistants, nurse practitioners, and pharmacists in the US, conducted in March 2018. To elicit HCP preferences for possible changes to the recommendation, the survey instrument included best-worst scaling (BWS), a stated-preference approach used in applications in health and health care [6, 7]. This study used object-case BWS, a type of BWS used to elicit preferences for a list of mutually exclusive objects, such as policy goals or recommendations. Conventional survey items were also included to assess knowledge, attitudes, and beliefs about adult vaccination.

\section{Survey Instrument}

The survey included questions designed to evaluate providers' knowledge of the ACIP recommendation, their attitudes and intentions toward offering pneumococcal vaccination to appropriate patients and toward possible changes in the recommendations, their preferences for possible changes in recommendation, and their beliefs about consequences of potential changes to recommendations.

After completing the items assessing knowledge of the ACIP recommendation, participants were shown the following information about the ACIP recommendation before they completed the survey questions related to possible changes in the recommendations:

"The ACIP/CDC currently recommends that a dose of 13-valent pneumococcal conjugate vaccine [PCV13 (Prevnar 13, Wyeth Pharmaceuticals, Inc., a subsidiary of Pfizer Inc.)] be followed by a dose of the 23-valent pneumococcal polysaccharide vaccine [PPSV23 (Pneumovax 23, Merck and Co., Inc.)] in all adults aged 65 years and older who have not previously received pneumococcal vaccine. A dose of Pneumovax 23 should be given $\geq 1$ year following a dose of Prevnar 13. The ACIP/ CDC has indicated that the recommendations for routine Prevnar 13 use among 
adults aged 65 years and older will be reevaluated in 2018 and revised as needed. We would like to know your opinion about a few possible outcomes of the ACIP/CDC re-evaluation in 2018. Please review the scenarios below. Please consider only the age-based recommendation for those aged 65 and older, and assume the other recommendations for pneumococcal vaccination (pediatric, immunocompromised, etc.) would remain the same."

Preferences for five possible ACIP recommendation scenarios for adult immunization against pneumococcal disease were assessed using the following options: (1) retain current recommendation (PCV13 and PPSV23 in series) with no planned re-evaluation; (2) retain current recommendation with a planned reevaluation; (3) change PCV13 to Category B; retain PPSV23 as Category A; (4) remove PCV13 from recommendation; retain PPSV23 as Category A; (5) remove all pneumococcal vaccines from the schedule. Each participant saw two BWS questions. The first presented all five of these scenarios, and participants were asked to identify their most preferred (best) and least preferred (worst) scenario (Fig. 1). The second presented the three remaining scenarios that were not selected as most or least preferred in the first question, and participants were again asked to select their most and least preferred recommendation from the presented scenarios. This process allowed for a full ranking of the five possible ACIP recommendation scenarios included in the study.

Finally, to understand potential consequences of changes to the recommendation, participants were told to imagine the ACIP had adopted one of the potential scenarios. The specific recommendation scenario was randomly assigned to the participant. The participant was then presented with a series of questions to explore his or her perceptions and beliefs about the consequences of the assigned potential recommendation.

Before data collection, the draft questionnaire was pretested in cognitive debriefing interviews $(N=8)$ within the target population to ensure that the items, response options, and recall periods were understandable and easily answered by survey participants. Cognitive pretest interviewing is a well-established qualitative research methodology used to identify problems with questionnaire items and response options [8].

\section{Participants}

Participants were identified and recruited through Survey Sampling International (SSI), a global panel provider and research partner for this study. SSI has access to panels of HCPs and patients who have agreed to participate in online research about health care-related issues and has recruited physicians and patients for published survey studies (e.g., [9-13]). SSI panel sources recruit HCPs from a variety of methods, including telephone or face-to-face via calling lists of hospitals and HCPs. HCPs are also recruited via online web registration following a two-step process. HCPs enroll in the panel, and their credentials are compared against lists of known HCPs (e.g., from the American Medical Association or National Provider Identifier) to validate the enrollee. A randomly selected sample of HCPs received an e-mail invitation to participate in an online survey programmed and hosted by SSI. Each e-mail invitation included a unique survey link and embedded password for accessing the survey as well as a unique random user identification number to prevent duplicate responses. No personal identifiers were stored directly with the survey responses. Via a web-based screener, participants answered eligibility questions about their health care specialty; whether they had a subspecialty (in which case they were not eligible); if they were pharmacists, whether they were licensed to administer immunizations; and whether they had prescribed, recommended, or administered a pneumococcal vaccination to an adult aged 65 years and older in the past 3 months. Participants also consented electronically to participate before completing the survey. Participants completing the survey were sent an electronic gift card equivalent to $\$ 85$. 


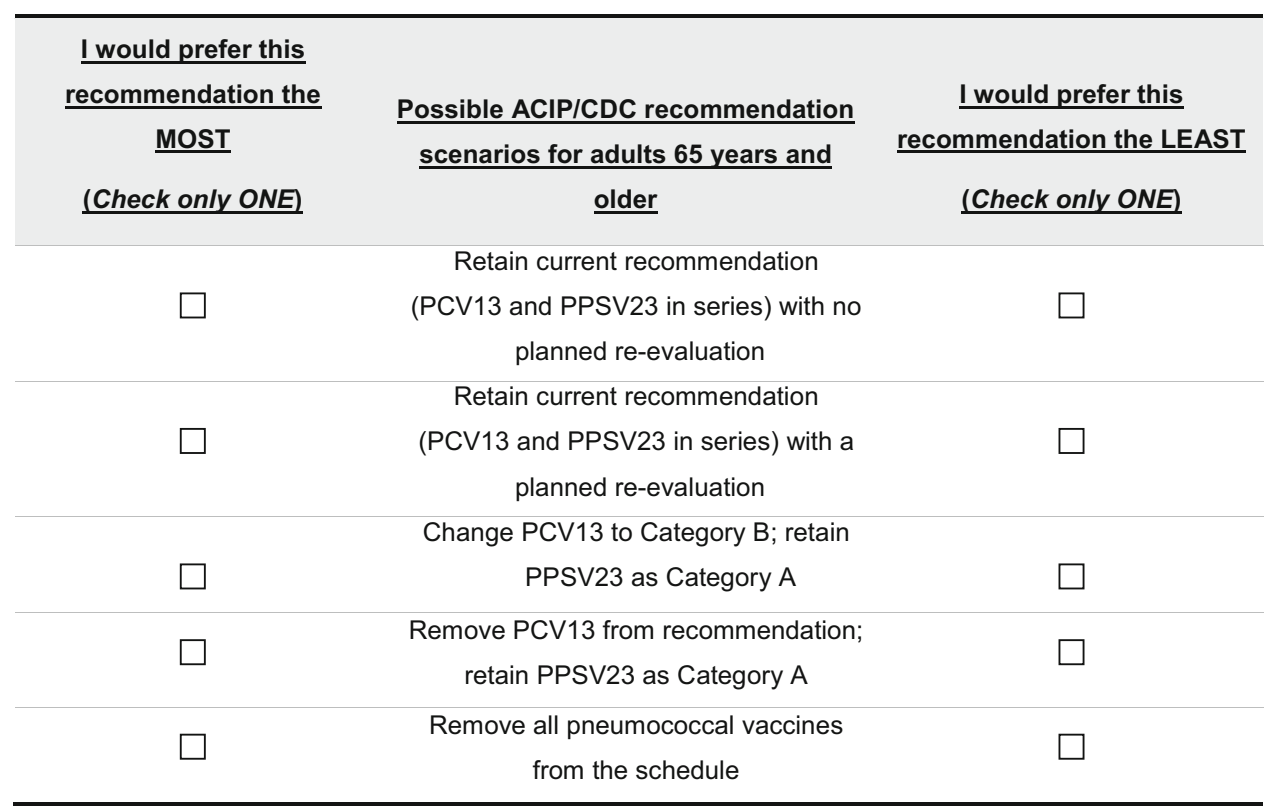

Fig. 1 Object-case BWS question. Example BWS question presenting five recommendation scenarios. ACIP Advisory Committee on Immunization Practices, $B W S$ best-worst

A sample of 1000 HCPs was planned. Recruitment targets focused on practitioners most likely to administer or prescribe pneumococcal vaccinations. To achieve balance among the types of HCP participating in the study, quotas of 700 physician participants (i.e., general practitioner, general internist, general internist/geriatrician, family medicine physicians), 100 physician assistant participants, 100 nurse practitioner participants, and 100 pharmacist participants were used. The study was reviewed by the RTI institutional review board and deemed exempt from full review.

\section{Statistical Analyses}

Analyses were performed on observed data only, and no imputation for missing values was performed. Questions that produced continuous data were summarized descriptively. Questions that produced categorical or ordinal data were summarized using frequencies and percentages, including the BWS items. The descriptive analyses were conducted using SAS Statistical Software, version 9.4 or higher [14]. scaling, $C D C$ Centers for Disease Control and Prevention, PCV13 13-valent pneumococcal conjugate vaccine, PPSV 23 23-valent pneumococcal polysaccharide vaccine

The BWS questions were further analyzed using best-minus-worst scores to obtain the relative importance weights across the sample and by HCP provider type. In each question, the item selected as "most preferred" was assigned a value equal to 1 , and the item selected as "least preferred" was assigned a value equal to -1 ; all other items were assigned a value equal to 0 . For each item, the assigned values were summed across questions and across participants and then divided by the number of times the item is presented in the BWS questions. The resulting scores indicate the order and the relative importance of each item to participants, such that larger positive values indicate a higher rank and greater relative desirability and larger negative values indicate a lower rank and greater relative undesirability. Items with values closer to 0 are relatively less important to participants than those with larger positive or negative values. To examine preference heterogeneity across provider type, we also calculated the scores separately by each HCP type in the sample. The BWS analyses were conducted using Sawtooth Software [15]. 


\section{RESULTS}

\section{Participants}

A total of 51,000 unique invitations were sent to HCPs to participate in the survey, to which 2961 HCPs responded and accessed the survey. Of the participants who responded, 808 were ineligible and 4 refused consent; 2149 HCPs remained eligible to participate in the survey. Among the 808 participants who were ineligible, $18 \%$ were not one of the HCPs of interest; $44 \%$ were ineligible because they indicated they had a subspecialty; $14 \%$ were ineligible because they were a pharmacist who was not certified to administer vaccinations; $24 \%$ were ineligible because they indicated they had not prescribed, recommended, or administered a pneumococcal vaccination to an adult aged 65 years and older in the past 3 months. One thousand HCPs completed the survey and were included in the analyses. The overall response rate was $2.0 \%$.

Participants' mean age was 44.7 years, $48.4 \%$ were female, and $48.3 \%$ described the geographic area of their practice as suburban (Table 1). Approximately half of physicians $(52.9 \%)$, physician assistants (56.0\%), and nurse practitioners $(52.0 \%)$ worked primarily in a private office practice; most pharmacists $(62.0 \%)$ worked in a retail pharmacy. HCPs saw approximately 50 patients/week aged 65 years and older and estimated that $83.8 \%$ of their patients aged 65 years and older are covered by private insurance, Medicaid, Medicare fee-forservice, or Medicare Advantage.

\section{SURVEY RESULTS}

\section{Attitudes and Beliefs Toward and Knowledge of Pneumococcal Vaccination Recommendation}

Among the overall sample of HCPs, 97.1\% indicated that it is extremely or somewhat important for adults aged 65 years and older to receive PCV13 in series with PPSV23, consistent with the then-current 2014 ACIP/CDC recommendation (Table S-1, supplemental appendix).
Virtually all participants indicated that they recommend pneumococcal vaccination for all adults aged 65 years and older (99.5\%) and follow the ACIP/CDC recommendation for pneumococcal vaccination in this population (98.5\%). Similarly, nearly all participants indicated that they are knowledgeable about the ACIP/CDC recommendation for pneumococcal vaccination for adults aged 65 years and older (98.9\%) and consider it important to stay informed about current ACIP/CDC recommendations for adult vaccination schedules (99.2\%).

Most HCPs correctly identified the recommendation and selected scenarios consistent with it when considering a hypothetical patient. When asked to select a statement that best represented the current recommendation for vaccination timing of the pneumococcal vaccine for adults aged 65 years and older, more than three-fourths of participants (78.4\%) selected the response consistent with the 2014 ACIP/CDC recommendation (Table S-2). Furthermore, a majority of the sample indicated that they would recommend a vaccination approach consistent with the ACIP/CDC recommendation for immunocompetent adults aged 65 years and older with no previous vaccination $(70.7 \%)$.

\section{Attitudes and Beliefs Toward Possible Changes to Recommendation}

Participants were asked if ACIP/CDC no longer recommended PCV13 in immunocompetent adults aged 65 years and older, how likely would the HCP be to stop recommending PCV13 to these individuals (Table S-3). Most HCPs $(88.9 \%)$ said they would likely stop recommending PCV13 to these individuals, and $97.0 \%$ of HCPs said they were likely to follow any new recommendations if there is another planned re-evaluation.

A total of $91.4 \%$ of participants reported that if ACIP/CDC were to revise the recommendation for pneumococcal vaccination, and adults aged 65 years and older had to pay out of pocket to cover the cost of the vaccination, they would likely spend time with these individuals to explain the importance of vaccination 
Table 1 Participant characteristics

\begin{tabular}{|c|c|c|c|c|c|}
\hline Category, $n(\%)$ & $\begin{array}{l}\text { Full sample } \\
(N=1000)\end{array}$ & $\begin{array}{l}\text { Physicians } \\
(n=700)\end{array}$ & $\begin{array}{l}\text { Physician assistants } \\
(n=100)\end{array}$ & $\begin{array}{l}\text { Nurse practitioners } \\
(n=100)\end{array}$ & $\begin{array}{l}\text { Pharmacists } \\
(n=100)\end{array}$ \\
\hline \multicolumn{6}{|l|}{ Gender $^{a}$} \\
\hline Female & $484(48.4 \%)$ & 277 (39.6\%) & $69(69.0 \%)$ & $91(91.0 \%)$ & $47(47.5 \%)$ \\
\hline Male & $512(51.3 \%)$ & $420(60.0 \%)$ & $31(31.0 \%)$ & $9(9.0 \%)$ & $52(52.5 \%)$ \\
\hline Other & $3(0.3 \%)$ & $3(0.4 \%)$ & 0 & 0 & $0^{\mathrm{a}}$ \\
\hline \multicolumn{6}{|l|}{ Age (in years) } \\
\hline Mean (SD) & 44.7 (11.39) & $46.6(11.04)$ & $38.0(10.21)$ & $45.1(10.76)$ & $38.0(10.55)$ \\
\hline Median & 44.0 & 46.0 & 36.0 & 43.5 & 35.0 \\
\hline Min, $\max$ & 22,84 & 26,84 & 22,72 & 26,69 & 24,67 \\
\hline Missing & 1 & 0 & 0 & 0 & 1 \\
\hline \multicolumn{6}{|l|}{ Years in practice } \\
\hline Mean (SD) & $13.8(9.88)$ & $15.2(10.02)$ & $9.7(7.40)$ & $10.7(8.86)$ & $11.0(9.75)$ \\
\hline Median & 12.0 & 14.0 & 8.0 & 8.0 & 8.0 \\
\hline Min, max & 0,58 & 1,58 & 0,34 & 1,40 & 1,38 \\
\hline \multicolumn{6}{|c|}{ Percentage of time spent in direct patient care } \\
\hline Mean $(S D)$ & $87.6(20.34)$ & $91.6(15.11)$ & $88.0(17.87)$ & $88.4(19.35)$ & $58.7(30.15)$ \\
\hline Median & 95.0 & 99.0 & 95.0 & 95.0 & 60.0 \\
\hline Min, $\max$ & 1,100 & 1,100 & 6,100 & 3,100 & 3,100 \\
\hline \multicolumn{6}{|c|}{ Geographic area of practice } \\
\hline Rural & $179(17.9 \%)$ & $115(16.4 \%)$ & $25(25.0 \%)$ & $23(23.0 \%)$ & $16(16.0 \%)$ \\
\hline Suburban & $483(48.3 \%)$ & $348(49.7 \%)$ & $41(41.0 \%)$ & $58(58.0 \%)$ & $36(36.0 \%)$ \\
\hline Urban & $338(33.8 \%)$ & $237(33.9 \%)$ & $34(34.0 \%)$ & $19(19.0 \%)$ & $48(48.0 \%)$ \\
\hline \multicolumn{6}{|c|}{ Primary work environment } \\
\hline $\begin{array}{l}\text { Private office } \\
\text { practice }\end{array}$ & $478(53.1 \%)$ & $370(52.9 \%)$ & $56(56.0 \%)$ & $52(52.0 \%)$ & - \\
\hline $\begin{array}{l}\text { Hospital-based } \\
\text { practice }\end{array}$ & $220(24.4 \%)$ & $191(27.3 \%)$ & $14(14.0 \%)$ & $15(15.0 \%)$ & - \\
\hline $\begin{array}{l}\text { Federally qualified } \\
\text { health center }\end{array}$ & $95(10.6 \%)$ & $70(10.0 \%)$ & $14(14.0 \%)$ & $11(11.0 \%)$ & - \\
\hline Urgent care & $45(5.0 \%)$ & $26(3.7 \%)$ & $11(11.0 \%)$ & $8(8.0 \%)$ & - \\
\hline $\begin{array}{l}\text { Integrated delivery } \\
\text { network }\end{array}$ & $42(4.7 \%)$ & $33(4.7 \%)$ & $3(3.0 \%)$ & $6(6.0 \%)$ & - \\
\hline $\begin{array}{l}\text { Long-term care } \\
\text { facility }\end{array}$ & $20(2.2 \%)$ & $10(1.4 \%)$ & $2(2.0 \%)$ & $8(8.0 \%)$ & - \\
\hline
\end{tabular}


Table 1 continued

\begin{tabular}{|c|c|c|c|c|c|}
\hline Category, $n$ (\%) & $\begin{array}{l}\text { Full sample } \\
(N=1000)\end{array}$ & $\begin{array}{l}\text { Physicians } \\
(n=700)\end{array}$ & $\begin{array}{l}\text { Physician assistants } \\
(n=100)\end{array}$ & $\begin{array}{l}\text { Nurse practitioners } \\
(n=100)\end{array}$ & $\begin{array}{l}\text { Pharmacists } \\
(n=100)\end{array}$ \\
\hline Retail pharmacy & - & - & - & - & $62(62.0 \%)$ \\
\hline Specialty pharmacy & - & - & - & - & $4(4.0 \%)$ \\
\hline $\begin{array}{l}\text { Home care } \\
\text { pharmacy }\end{array}$ & - & - & - & - & $1(1.0 \%)$ \\
\hline Hospital & - & - & - & - & $25(25.0 \%)$ \\
\hline $\begin{array}{l}\text { Federally qualified } \\
\text { health center }\end{array}$ & - & - & - & - & $4(4.0 \%)$ \\
\hline Urgent care & - & - & - & - & 0 \\
\hline $\begin{array}{l}\text { Integrated delivery } \\
\text { network }\end{array}$ & - & - & - & - & $2(2.0 \%)$ \\
\hline $\begin{array}{l}\text { Long-term care } \\
\text { facility }\end{array}$ & - & - & - & - & $2(2.0 \%)$ \\
\hline
\end{tabular}

(Table S-4). Slightly more than half $(57.1 \%)$ of the sample responded they thought that adults aged 65 years and older would likely pay out of pocket for a pneumococcal vaccination. However, three-fourths of the sample (76.1\%) reported they would likely limit recommending, prescribing, and administering pneumococcal vaccinations depending on the individual's ability to pay out of pocket if ACIP/ CDC were to revise the recommendation such that Medicare and commercial insurance no longer covered the cost of the vaccinations.

\section{Preferences for Possible Changes to the Recommendation}

Using HCPs' responses to the BWS questions, the frequency with which each possible ACIP recommendation scenario was selected as most and least preferred was calculated (Table 2). More than half of participants $(52.6 \%)$ most preferred the option to retain the recommendation with a planned re-evaluation, followed by the options to retain without planning a reevaluation (40.0\%). The least-preferred option for nearly all participants (87.3\%) was to remove all pneumococcal vaccines from the schedule.

Figure 2 presents the relative importance weights of the full sample relative to the reference item "Retain current recommendation with a planned re-evaluation." This analysis indicated retaining the then-current (2014) recommendation with a scheduled reevaluation was 1.3 times more preferred (10/ $7.6=1.3$ ) by HCPs than retaining the thencurrent recommendation with no planned reevaluation. Removing both pneumococcal vaccinations from the adult schedule was the leastpreferred recommendation relative to the other alternatives.

\section{Anticipated Changes to Practice Under Potential Recommendation Scenarios}

Each participant was randomly assigned to one of four of the five hypothetical ACIP recommendation scenarios (excluding the recommendation to retain the current recommendation with no planned re- 
Table 2 Frequency of ACIP/CDC recommendation chosen in the first best-worst scaling question

\begin{tabular}{lll}
\hline Possible ACIP/CDC recommendations & $\begin{array}{l}\text { Frequency chosen } \\
\text { least preferred }\end{array}$ & $\begin{array}{c}\text { Frequency chosen } \\
\text { most preferred }\end{array}$ \\
\hline $\begin{array}{l}\text { Retain current recommendation (PCV13 and PPSV23 in series) } \\
\text { with no planned re-evaluation }\end{array}$ & $45(4.5 \%)$ & $400(40.0 \%)$ \\
$\begin{array}{l}\text { Retain current recommendation (PCV13 and PPSV23 in series) } \\
\text { with a planned re-evaluation }\end{array}$ & $32(3.2 \%)$ & $526(52.6 \%)$ \\
$\begin{array}{l}\text { Change PCV13 to Category B; retain PPSV23 as Category A } \\
\text { Remove PCV13 from recommendation; retain PPSV23 } \\
\text { as Category A }\end{array}$ & $21(2.1 \%)$ & $32(3.2 \%)$ \\
Remove all pneumococcal vaccines from the schedule & $29(2.9 \%)$ & $37(3.7 \%)$ \\
\end{tabular}

ACIP Advisory Committee on Immunization Practices, CDC Centers for Disease Control and Prevention, PCV13 13-valent pneumococcal conjugate vaccine, PPSV23 23-valent pneumococcal polysaccharide vaccine

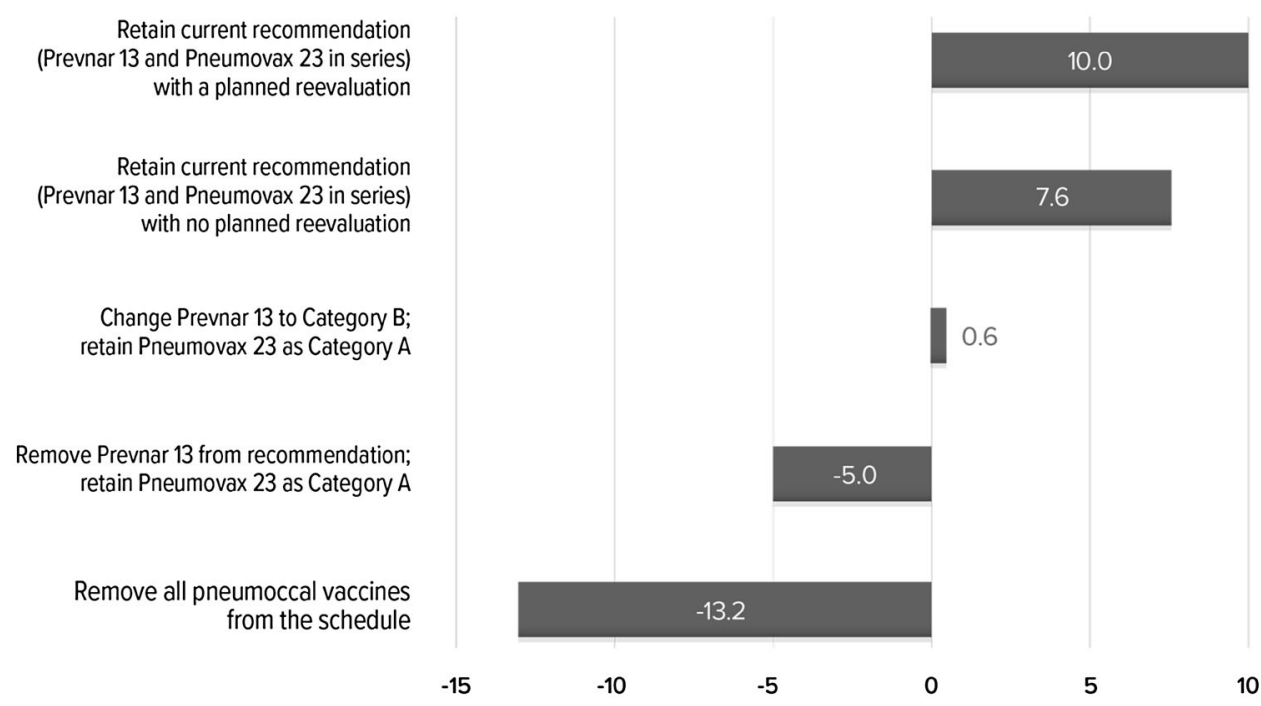

Fig. 2 Best-worst scaling relative importance weights $(N=1000)$. Larger positive values indicate greater relative desirability. Items with values closer to 0 are relatively less important to participants than those with larger positive or negative values. Retaining the then-current (2014) recommendation (PCV13 and PPSV23 in series) with a planned

evaluation) and asked to rate their level of agreement (strongly agree, agree, disagree, strongly disagree) with a variety of statements related to anticipated consequences on their practice due to implementing that possible recommendation (Figs. 3 and 4; Figures S-1 and $\mathrm{S}-2)$. re-evaluation was 1.3 times more preferred (10/7.6) than retaining the then-current recommendation with no planned re-evaluation and 16.7 times more preferred $(10 / 0.6=16.7)$ than changing PCV13 to a category B and retaining PPSV23 as a category A

For the option to retain the recommendation with a planned re-evaluation, most HCPs agreed that it would be easier to track whether patients had been fully immunized (79.6\%) and easier to comply with the revised recommendation $(78.2 \%) ; 85.9 \%$ would be comfortable implementing the recommendation in their practices 
A

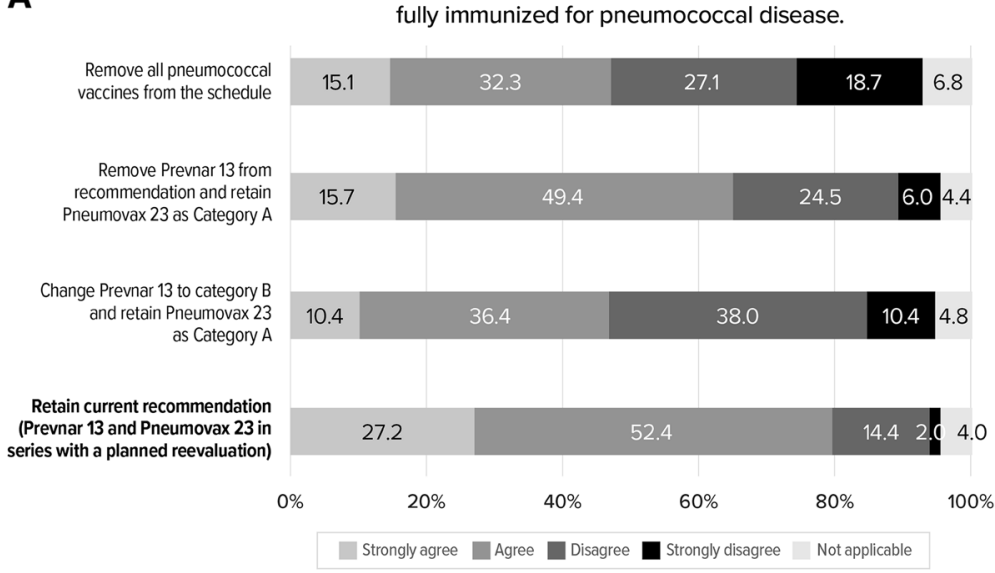

B

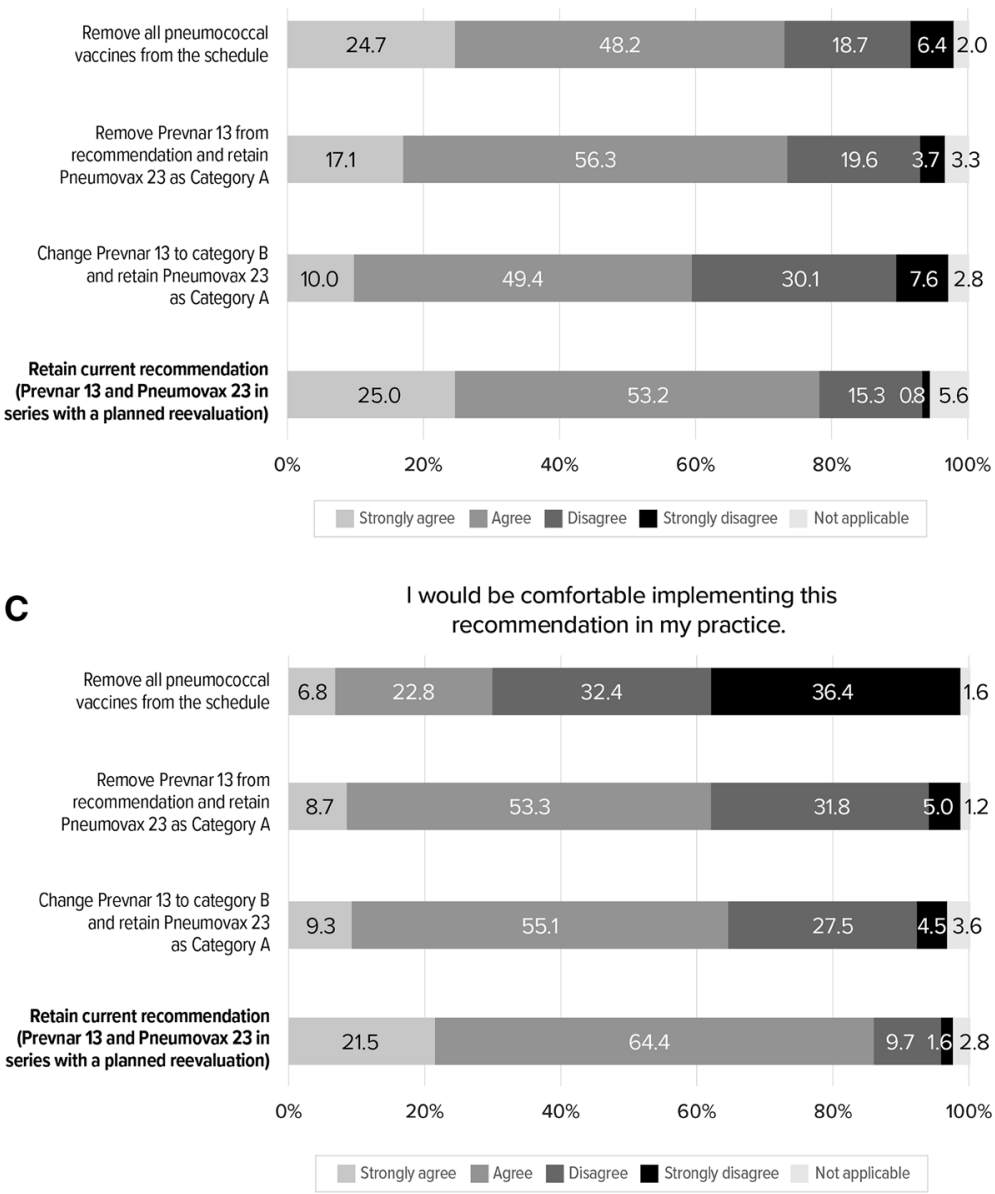

Fig. 3 Agreement with anticipated benefits of potential changes to ACIP/CDC Recommendation: percentage of participants selecting each response option. Response options in bold are consistent with the then-current (2014) ACIP/CDC recommendation. ACIP Advisory
Committee on Immunization Practices, $C D C$ Centers for Disease Control and Prevention, PCV13 13-valent pneumococcal conjugate vaccine, PPSV23 23-valent pneumococcal polysaccharide vaccine 
A

A

Remove all pneumococcal
vaccines from the schedule
Remove Prevnar 13 from
recommendation and retain
Pneumovax 23 as Category A
Change Prevnar 13 to category B
and retain Pneumovax 23
as Category A
Retain current recommendation
(Prevnar 13 and Pneumovax 23 in
series with a planned reevaluation)

B

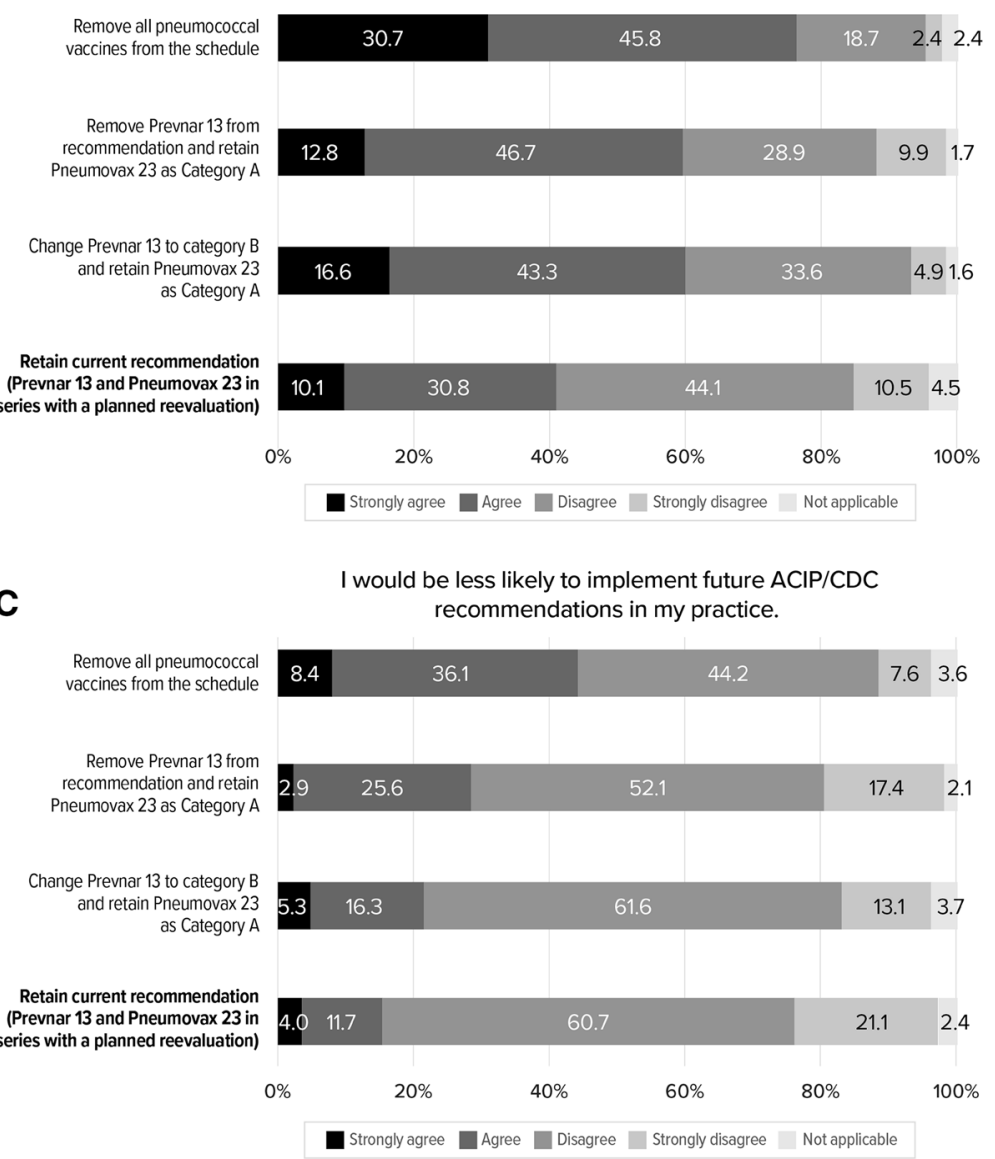

I would be confused by the change as the adult pneumococcal vaccination recommendation was updated in 2014.

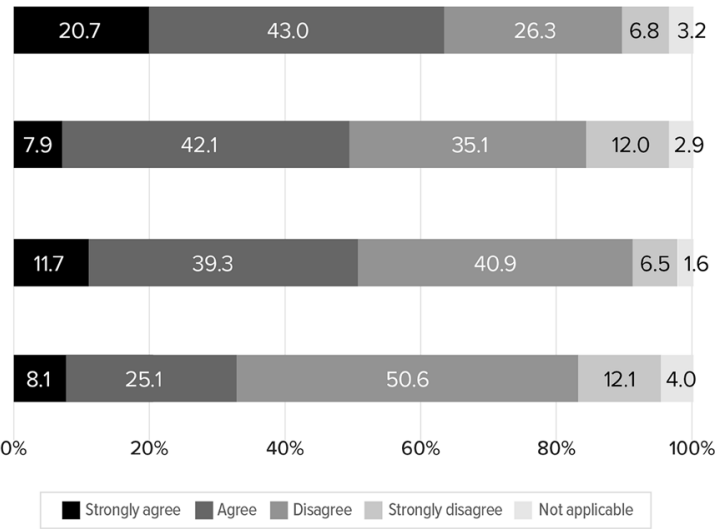

This change to the recommendation would increase hesitancy and/or suspicion about vaccines among my patients.

$0 \%$

Strongly agree $\square$ Agree $\square$ Disagree $\square$ Strongly disagree $\square$ Not applicable $\%$
Fig. 4 Agreement with anticipated challenges of potential changes to ACIP/CDC recommendation: percentage of participants selecting each response option. Response options in bold are consistent with the then-current (2014) ACIP/CDC recommendation. ACIP Advisory 
(Fig. 3). For this same option, $49.6 \%$ of HCPs agreed that more of their patients would refuse vaccination and $64.6 \%$ agreed that it would be more burdensome to counsel patients on the importance of pneumococcal vaccination if the patient would face additional out-of-pocket expense (Figure S-2). Furthermore, $40.9 \%$ of HCPs indicated that the option to retain the current recommendation with a planned reevaluation would increase hesitancy and/or suspicion about vaccines among patients, and $15.7 \%$ of HCPs indicated that this option would make them less likely to implement future ACIP/CDC recommendations (Fig. 4).

\section{DISCUSSION}

This study aimed to assess HCP preferences for potential changes to the 2014 ACIP/CDC pneumococcal immunization recommendation among adults aged 65 years and older as well as associated knowledge, attitudes, and beliefs. The HCPs surveyed were involved in recommending, prescribing, or administering pneumococcal vaccine; were knowledgeable about and claim to follow the recommendation; and consider it important to keep up with recommendations as they are revised and updated.

HCPs preferred retaining the 2014 recommendation relative to the alternatives presented, with a slight preference among HCPs for retaining PCV13 and PPSV23 in series with a planned re-evaluation relative to retaining the recommendation indefinitely. Removing both pneumococcal vaccinations from the adult schedule was the least-preferred recommendation, with the potential to negatively impact access to vaccines and cause disparity among potential recipients. The option of switching PCV13 to category B while retaining PPSV23 as category A-the equivalent of the shared clinical decision making endorsed by the committee in June 2019, during revision of this articlewas less preferred to retaining a category A (i.e., routine) recommendation but preferred relative to the option of removing the PCV13 recommendation while retaining PPSV23. There was no difference in the order and very little, if any, difference in preferences when qualitatively compared across HCP type.

The current study provides further support for the role of perceived reimbursement challenges as a barrier to the use of pneumococcal vaccines in adults $[16,17]$. A survey conducted in December 2015 and January 2016 evaluating 617 US primary care physicians' perceptions and knowledge of the ACIP recommendations for pneumococcal vaccination found most HCPs were well informed about the pneumococcal recommendation, but identified reimbursement challenges-including insufficient coverage of vaccine cost, lack of coverage by private insurance or Medicare/Medicaid, and lack of coverage if vaccines were not administered according to schedule-as barriers to following ACIP guidelines for pneumococcal vaccination [16]. Many HCPs in this study also indicated concerns about reimbursement, despite a reported $84 \%$ of their patients aged 65 years and older being covered by private insurance, Medicaid, or a Medicare program, which generally cover these vaccinations and should allow for administration by HCPs with no outof-pocket expense to the patient.

The 2015-2016 survey found some confusion regarding which pneumococcal vaccine should be administered first, which was also noted in the current study despite being fielded 2 years later [16]. There may be an opportunity for more frequent and effective continuing education and dissemination of relevant knowledge so that HCPs have the information they need in a medium that is most useful and valuable to them. This seems like a particularly important consideration in the context of shared clinical decision making, as a survey conducted among pediatricians and family physicians in late 2016 documented misconceptions and confusion regarding category $\mathrm{B}$ recommendations [18]. Responses to items in the current study around ease of tracking full pneumococcal immunization and ease of complying with the revised recommendation in the hypothetical scenario of changing PCV13 to a category B while retaining PPSV23 as category A suggest that this is likely to be a challenge in the implementation of the new recommendation (Fig. 3a, b respectively). 
Most HCPs indicated that they are likely to spend time with their older adult patients to explain the importance of pneumococcal vaccination, though approximately $43 \%$ of HCPs think these adults are not at all likely to pay out of pocket for a pneumococcal vaccination dose. Surprisingly, if Medicare and commercial insurance no longer covered the cost of these vaccinations, approximately three-quarters of HCPs were likely to limit recommending, prescribing, and administering pneumococcal vaccinations, depending on the patient's ability to pay for the out-of-pocket expense. These results suggest a potential for health inequities if the ACIP/CDC revise the recommendation such that insurance coverage is impacted by the changes, or indeed if HCPs mistakenly believe the change in type of recommendation changes reimbursement, as was documented by Kempe and colleagues [18]. This could result in systematic differences in the health status of different populations based on their ability to access and pay for pneumococcal vaccinations.

Some strengths and limitations of this study should be considered. This study had limited inclusion/exclusion criteria and enrolled a heterogeneous sample of 1000 HCPs who prescribe and administer pneumococcal vaccines. The sample achieved diversity across practice type and settings and reasonable geographic distribution of participants practicing in urban and suburban areas. The use of cognitive debriefing interviews to pretest the survey questionnaire to ensure that the items, response options, and recall periods were understandable and easily answered by survey participants is an important strength. A potential limitation of the study is that few HCPs who practiced primarily in urgent care centers, integrated delivery networks, or long-term care facilities participated in the study and may have been underrepresented, although data on the distribution of HCPs' practice environments in these practice settings were not found in the published literature. HCPs working in these environments may have responded differently to survey questions, a possibility not assessed in the current study. Similarly, $17.9 \%$ of HCPs were practicing in rural areas, and the results may have been different with a larger number of
HCPs working primarily in a rural community if preferences, attitudes, or intentions vary according to the urbanicity of the work environment. Nevertheless, in terms of distribution among rural, suburban, and urban areas, the physician subsample in our study was generally comparable to the sample of physicians who participated in the Vaccine Policy Collaborative Initiative, which was considered to be representative of the American College of Physicians and the American Academy of Family Physicians memberships [16].

Another potential limitation is awareness of the rationale underlying the ACIP re-evaluation of the recommendation among the participants. The survey did not present the rationale or assess awareness of it. It is possible that HCP preferences may be influenced by such knowledge. Likewise, the survey was fielded in March 2018, and preferences of physicians may have been affected by relevant evidence disseminated since.

An additional limitation of this study, and of all voluntary surveys, is potential responder bias, which may lead to inaccurate results. Study results are also based on self-report, and there is no way to corroborate the survey data. There is also some potential for bias as some participants may have prepared for the survey by reviewing the current ACIP/CDC recommendation before-or while-completing the survey, inflating the proportion of HCPs who knew the ACIP recommendation. Selection bias is a potential limitation of this study because participants were recruited through a panel. Thus, the HCP participants may not be representative of HCPs in the US, and the physician subsample in the current survey was younger than in the survey study conducted by the Vaccine Policy Collaborative Initiative sample [16], potentially limiting the generalizability of the results. Furthermore, the preferences of those who participate in a panel may be systematically different from those who do not participate in a panel. In addition, the characteristics and preferences of those potential participants who chose to complete the survey may be systematically different from those who did not, and there is no way to measure or control for these potential biases, though it is not 
apparent whether such a bias would be correlated with preferences for pneumococcal vaccine recommendations. Finally, the attitudes and intentions measured in the survey are in response to hypothetical scenarios and may differ from the participants' actual behavior.

\section{CONCLUSIONS}

Almost all HCPs surveyed preferred to retain the 2014 ACIP/CDC recommendation pneumococcal vaccination among adults aged 65 years and older, with a slight preference for including a planned re-evaluation rather than retaining it indefinitely, while few preferred changing PCV13 to a category B recommendation while retaining PPSV23 as category $\mathrm{A}$, the option endorsed by the ACIP in June of 2019. HCPs' preferences for the ACIP/CDC recommendation reflect potential concerns about the increased potential for health inequities if the ACIP/CDC revise recommendations such that insurance coverage is impacted by the changes.

\section{ACKNOWLEDGEMENTS}

We thank the participants of this study.

Funding. This study, preparation of this article, and the Rapid Service Fees were funded by Pfizer Inc. All authors had full access to all of the data in this study and take complete responsibility for the integrity of the data and accuracy of the data analysis.

Medical Writing, Editorial, and Graphics Assistance. Kate Lothman of RTI Health Solutions provided medical writing assistance. Ryan Miller of RTI Health Solutions provided graphic design services, and Paul Hobson of RTI Health Solutions provided editorial services. These services were funded by Pfizer Inc.

Authorship. All named authors meet the International Committee of Medical Journal Editors (ICMJE) criteria for authorship for this article, take responsibility for the integrity of the work as a whole, and have given their approval for this version to be published.

Disclosures. This study was conducted under a research contract between RTI Health Solutions and Pfizer Inc. and was funded by Pfizer Inc. Kelly Hollis is a salaried employee of RTI Health Solutions. Kelley Myers is a salaried employee of RTI Health Solutions. Christine Poulos is a salaried employee of RTI Health Solutions. Patricia Sacco is a salaried employee of RTI Health Solutions. Carolyn Sweeney is a salaried employee of RTI Health Solutions. Vincenza Snow is a salaried employee of Pfizer Inc. Jeff Vietri is a salaried employee of Pfizer Inc.

Compliance with Ethical Guidelines. The study was reviewed by the RTI institutional review board and deemed exempt from full review. All participants provided electronic informed consent.

Data Availability. The datasets generated during and/or analyzed during the current study are available from the corresponding author on reasonable request.

Open Access. This article is distributed under the terms of the Creative Commons Attribution-NonCommercial 4.0 International License (http://creativecommons.org/licenses/ by-nc/4.0/), which permits any noncommercial use, distribution, and reproduction in any medium, provided you give appropriate credit to the original author(s) and the source, provide a link to the Creative Commons license, and indicate if changes were made.

\section{REFERENCES}

1. Tomczyk S, Bennett NM, Stoecker C, et al. Use of 13 -valent pneumococcal conjugate vaccine and 23-valent pneumococcal polysaccharide vaccine among adults aged $\geq 65$ years: recommendations of the Advisory Committee on Immunization Practices (ACIP). MMWR Morb Mortal Wkly Rep. 2014;63:822-5. 
2. National Foundation for Infectious Diseases [Internet]. Pneumococcal disease. http://www. adultvaccination.org/pneumococcal_vaccine_ vaccination_adult_immunization.htm Accessed 5 Jul 2017.

3. Kobayashi M, Bennett NM, Gierke R, et al. Intervals between PCV13 and PPSV23 vaccines: recommendations of the Advisory Committee on Immunization Practices (ACIP). MMWR Morb Mortal Wkly Rep. 2015;64(34):944-7.

4. Centers for Disease Control and Prevention (CDC) [Internet]. Intervals Between PCV13 and PPSV23 Vaccines: Recommendations of the Advisory Committee on Immunization Practices (ACIP). https:// www.cdc.gov/mmwr/preview/mmwrhtml/ mm6434a4.htm Accessed 5 Jun 2019.

5. Centers for Disease Control and Prevention (CDC) [Internet]. ACIP Evidence to Recommendations Framework. https://www.cdc.gov/vaccines/acip/ recs/grade/downloads/ACIP-evidence-rec-frame508.pdf Accessed 9 Nov 2018.

6. Louviere JJ, Flynn TN, Marley AAJ. Best-worst scaling: theory, methods, and applications. Cambridge: Cambridge University Press; 2015.

7. Medical Device Innovation Consortium (MDIC) [Internet]. A framework for incorporating information on patient preferences regarding benefit and risk into regulatory assessments of new medical technology. http://mdic.org/spi/pcbr-frameworkreport-release/framework-report/ Accessed 15 Oct 2017.

8. Groves R, Fowler F, Couper M, et al. Survey methodology. 2nd ed. Hoboken: Wiley; 2009.

9. Hauber AB, Mange B, Price MA, et al. Administration options for pegfilgrastim prophylaxis: patient and physician preferences from a cross-sectional survey. Support Care Cancer. 2018;26(1):251-60.
10. Hauber AB, Obi EN, Price MA, et al. Quantifying the relative importance to patients of avoiding symptoms and outcomes of heart failure. Curr Med Res Opin. 2017;4:1-12.

11. Marion S, Tzivelekis S, Darden C, et al. "Same-day" administration of pegfilgrastim following myelosuppressive chemotherapy: clinical practice and provider rationale. Support Care Cancer. 2016;24(9):3889-96.

12. Magrey M, Bozyczko M, Wolin D, et al. A pilot study to assess the feasibility of a web-based survey to examine patient-reported symptoms and satisfaction in patients with ankylosing spondylitis receiving secukinumab. Drugs Real World Outcomes. 2019;6(2):83-91.

13. Botha W, Donnolley N, Shanahan M, et al. Societal preferences for fertility treatment in Australia: a stated preference discrete choice experiment. J Med Econ. 2018;15:1-23.

14. SAS Statistical Software, version 9.4. SAS Institute, Inc., Cary, USA.

15. Sawtooth Software. The MaxDiff/Web System technical paper, version 8. Sawtooth Software, Inc., Orem, USA.

16. Hurley LP, Allison MA, Pilishvili T, et al. Primary care physicians' struggle with current adult pneumococcal vaccine recommendations. J Am Board Fam Med. 2018;31(1):94-104.

17. Kempe A, Hurley L, Stokley S, et al. Pneumococcal vaccination in general internal medicine practice: current practice and future possibilities. J Gen Intern Med. 2008;23(12):2010-3.

18. Kempe A, Allison MA, MacNeil JR, et al. Knowledge and attitudes regarding category B ACIP recommendations among primary care providers for children. Acad Pediatr. 2018;18(7):763-8. 